\title{
3D-QSAR Study for Checkpoint Kinase 2 Inhibitors through Pharmacophore Hypotheses
}

\author{
Yen-Ling Wang, Chun-Yuan Lin, Kuei-Chung Shih, and Chuan-Yi Tang
}

\begin{abstract}
DNA-damage is induced by ionizing radiation, genotoxic chemicals or collapsed replication forks. To prevent and repair the DNA-damage, mammalian cells will control and stabilize the genome by cell cycle checkpoint. Checkpoint kinase 2 (Chk2) is one of the important kinases that has a great effect on DNA-damage and plays an important role in response to DNA double-strand breaks and related lesions. Hence, in this study, we will concentrate on Chk2 and the purpose is to build the pharmacophore hypotheses (PhModels) by 3D-QSAR study which can identify inhibitors with high biological activities. Ten PhModels were generated by the HypoGen Best algorithm. The established PhModel, Hypo01, was evaluated in the cost function analysis of its correlation coefficient $(r)$, RMS, cost difference, and configuration cost, with the values: $0.955,1.28$, 192.51, and 16.07, respectively. The result of Fischer's cross-validation test for Hypo01 model yielded a $95 \%$ confidence level, and the correlation coefficient $\left(r_{\text {test }}\right)$ of the testing set yielded a best value of 0.81 .
\end{abstract}

Index Terms-CHK2, drug design, 3D-QSAR, pharmacophore.

\section{INTRODUCTION}

DNA-damage is induced by ionizing radiation, genotoxic chemicals or collapsed replication forks, and when DNA was damaged or the responses of cells were failure, the mutation associated with the breast or ovarian cancer of genes may occur. To prevent and repair the DNA-damage, mammalian cells will control and stabilize the genome by cell cycle checkpoint. The checkpoint pathway consists of several kinases, such as ataxia telangiectasia mutated protein (ATM $[1],[2]$ ), ataxia telangiectasia and Rad3-related protein (ATR [1], [2]), Checkpoint kinase 1 (Chk1 [3], [4]), and Checkpoint kinase 2 (Chk2 [5]-[8]).

ATM and ATR are upstream kinases passing messages to downstream kinases and phosphorylating several proteins that initiate activation of the DNA-damage checkpoint. Moreover, ATM is a primarily pathway to activate p53 (protein 53 [9]) by Chk2, and ATR may influence the phosphorylation of Chk1. Both Chk1 and Chk2 are key

Manuscript received January 15, 2013; revised March 6, 2013. Part of this work was supported by the National Science Council (NSC) with a grant of project contact NSC100-2221-E-182-057-MY3 and by Chang Gung University under Grant UERPD290101. We are grateful to the National Center for High-Performance Computing (NCHC) for computer time and facilities.

Yen-Ling Wang and Chun-Yuan Lin are with the Chang Gung University, Department of CSIE, Taoyuan 33302, Taiwan (e-mail: M0029009@stmail.cgu.edu.tw, cyulin@mail.cgu.edu.tw).

Kuei-Chung Shih and Chuan-Yi Tang are with the National Tsing Hua University, Department of Computer Science, Hsinchu 30013, Taiwan (e-mail: shiuh0307@gmail.com, cytang@pu.edu.tw). components in DNA-damage; however their cellular activities are different. Chk1 is involved in S and G2 phases of the cell cycle with ATR pathway. By contrast, Chk2 is activated in all phases through ATM-dependent pathway and plays an important role in response to DNA double-strand breaks and related lesions. Furthermore, Chk1 is an unstable protein and lacks the forkhead-associated domain (FHA) which was involved in several processes that protect against cancer and can be found in Chk2. Therefore, we will concentrate on Chk2 in this study.

Recently, some studies identified the inhibitors of Chk2 [10]-[14], and they also showed the crystal structures of Chk2 complex. They are selective, reversible, and ATP-competitive Chk2 inhibitors demonstrated that they effectively restrain the radiation-induced phosphorylation of Chk2. In addition, several selective Chk2 inhibitors have also been was identified and the researches indicated that they are potent and selective inhibitors of Chk2 with chemotherapeutic and radio-sensitization potential. On structure-based drug design, several developments of Chk2 were published.

Quantitative structure-activity relationship model (QSAR model) is a regression or classification model, and is an important technique in the rational drug design. It is used to correlate the structure properties of compounds with their biological activities. The method to predict the quality by QSAR was improved by considering the three-dimensional structure QSAR (3D-QSAR) [15]-[20] of targeted inhibitor. Therefore, the compound structure can be directly optimized in the $3 \mathrm{D}$ space. The comparative molecular field analyses (CoMFA) [21]-[26] and the comparative molecular similarity indices analyses (CoMSIA) [22]-[28] for Chk2 inhibitors were performed by ligand-based and receptor-guided alignment. They used the co-crystal structure from protein data bank (PDB code: 2CN8) [7], and then they identified new plausible binding modes used as template for 3D-QSAR [22]. There is another research of Chk2 studied in QSAR/QSPR [29] providing structures that will improve reducing the side effects of Chk2 inhibitors.

Pharmacophore [16]-[20], [30]-[32] is a set of structural features responsible for its biological activity of a molecule. It allowed compounds with diverse structures to find the common chemical features by ligand pharmacophore mapping, and that is different from CoMFA and CoMSIA. Thus, pharmacophore can explain how diverse ligands bind to a receptor site by these features, and visualize the feature of potential chemical interactions between ligands and receptors. Moreover, pharmacophore can easily and quickly identify candidate inhibitors for a target protein based on 3D query. Therefore, in this study, the purpose is to use 
3D-QSAR study to build Pharmacophore hypotheses (denoted as PhModels) for Chk2 inhibitors. We have faith that PhModels with the information about potential chemical interactions can help medicinal chemists to identify or design new Chk2 inhibitors

TABLE I: EXPERIMENTAL AND ESTIMATED IC ${ }_{50}$ VALUES OF THE TRAINING-SET INHIBITORS FOR CHK2

\begin{tabular}{|c|c|c|c|}
\hline No. & $\begin{array}{c}\text { Experimental } \mathrm{IC}_{50} \\
(\mathrm{nM})\end{array}$ & Estimated IC $_{50}(\mathrm{nM})$ & Error $^{\mathrm{a}}$ \\
\hline 1 & 2.3 & 15 & +6.6 \\
\hline 2 & 6.6 & 6.8 & +1 \\
\hline 3 & 14 & 20 & +1.4 \\
\hline 4 & 18 & 8.5 & -2.1 \\
\hline 5 & 23 & 19 & -1.2 \\
\hline 6 & 37 & 30 & -1.2 \\
\hline 7 & 47 & 23 & -2.1 \\
\hline 8 & 70 & 110 & +1.6 \\
\hline 9 & 72 & 47 & -1.5 \\
\hline 10 & 110 & 220 & +1.9 \\
\hline 11 & 270 & 670 & +2.5 \\
\hline 12 & 470 & 2200 & +4.7 \\
\hline 13 & 640 & 2000 & +3.1 \\
\hline 14 & 830 & 1200 & +1.5 \\
\hline 15 & 900 & 1000 & +1.1 \\
\hline 16 & 1100 & 970 & -1.1 \\
\hline 17 & 1200 & 1100 & -1.1 \\
\hline 18 & 1800 & 860 & -2.1 \\
\hline 19 & 6700 & 1700 & -3.9 \\
\hline 20 & 15000 & 22000 & +1.5 \\
\hline 21 & 19000 & 3600 & -5.3 \\
\hline 22 & 28000 & 6800 & -4.1 \\
\hline 23 & 37000 & 63000 & +1.7 \\
\hline 24 & 50000 & 16000 & -3.2 \\
\hline 25 & 100000 & 160000 & +1.6 \\
\hline
\end{tabular}

TABLE II: CHARACTERISTICS OF THE TEN PHARMACOPHORE HYPOTHESES FOR CHK2

\begin{tabular}{|c|c|c|c|c|c|}
\hline No. & $\begin{array}{c}\text { Total } \\
\text { cost }\end{array}$ & $\begin{array}{c}\text { Cost } \\
\text { difference }\end{array}$ & $\begin{array}{c}\text { RMS } \\
\text { deviation }\end{array}$ & $\begin{array}{c}\text { Training } \\
\text { set }(\boldsymbol{r})\end{array}$ & $\begin{array}{c}\text { Testing } \\
\text { set }\left(\boldsymbol{r}_{\text {test }}\right)\end{array}$ \\
\hline $\mathbf{1}$ & $\mathbf{1 1 1 . 6 8}$ & $\mathbf{1 9 2 . 5 1}$ & $\mathbf{1 . 2 8}$ & $\mathbf{0 . 9 5 5}$ & $\mathbf{0 . 8 1 0}$ \\
\hline 2 & 117.68 & 186.51 & 1.46 & 0.941 & 0.772 \\
\hline 3 & 121.50 & 182.69 & 1.59 & 0.929 & 0.769 \\
\hline 4 & 122.78 & 181.41 & 1.62 & 0.927 & 0.771 \\
\hline 5 & 122.98 & 181.21 & 1.63 & 0.926 & 0.807 \\
\hline 6 & 123.14 & 181.05 & 1.59 & 0.929 & 0.777 \\
\hline 7 & 125.01 & 179.18 & 1.65 & 0.924 & 0.802 \\
\hline 8 & 125.36 & 178.83 & 1.69 & 0.920 & 0.663 \\
\hline 9 & 125.66 & 178.53 & 1.69 & 0.919 & 0.794 \\
\hline 10 & 125.89 & 178.30 & 1.69 & 0.920 & 0.795 \\
\hline
\end{tabular}

a (null cost - total cost), null cost $=304.19$, fixed cost $=89.77$, configuration cost $=16.07$. All costs are in units of bits.

\section{MAterials AND MethodS}

\section{A. Biological Data Collection}

In order to construct the PhModels, at first, we collected the Chk2 inhibitors with two-dimensional structures and the biological activity values from the ChEMBL database [33]. Then, according to the structure variations and chemical differences in the kinase inhibitor activity, 158 known Chk2 inhibitors were selected and retrieved. The biological activity of 158 known Chk2 inhibitors was represented as $\mathrm{IC}_{50}$ (nanomolar, $\mathrm{nM}$ ).

\section{B. Designing a Pharmacophore Approach}

Before generating the PhModels, we should divide the 158 Chk2 inhibitors into the training set and testing set, respectively. The rules used to select training set inhibitors are according to the following: (1) All selected compounds should have clear and concise information including structure features and activity range. (2) At a minimum, 16 diverse compounds for training-set were selected to ensure the statistical significance. (3) The training-set should contain the most and the least active compounds. (4) The biological activities of the compounds spanned at least 4 orders of magnitude. Based on the above four rules, the 158 Chk2 inhibitors were divided.

The training set is used to construct the PhModels, and the $\mathrm{IC}_{50}$ values of these 25 inhibitors are ranged from 2.3 to $100,000 \mathrm{nM}$ (Table I). The testing set remaining 133 inhibitors with the chemical structures and $\mathrm{IC}_{50}$ values shown is used to test the predictive ability of the PhModels, and the $\mathrm{IC}_{50}$ values of the 133 testing set inhibitors are ranged from 3.4 to $74,000 \mathrm{nM}$. After selecting the training set inhibitors, we established ten PhModels at first, and then we used the cost function and Fischer's cross-validation test to estimate the prediction abilities of PhModels.

\section{Pharmacophore Generation}

In this study, we used the HypoGen program [34] in Accelrys Discovery studio 2.1 to generate PhModels. At the initial step, 3D conformations of the training set inhibitors were generated by using "3D-QSAR Pharmacophore Generation protocol" with the BEST generating algorithm and based on the CHARMm-like force field. The CHARMm-like force field in conjunction with three rules: (1) Conjugate-gradient minimization in torsion space. (2) Conjugate-gradient minimization in Cartesian space. (3) Quasi-Newton minimization in Cartesian space. The conformational-space energy was constrained $\leq 20 \mathrm{kcal} / \mathrm{mol}$ which represented the maximum allowed energy above the global minimum energy. For each training set inhibitor, the number of the diverse $3 \mathrm{D}$ conformations was set to $\leq 255$. All other parameters were set as default values. Following the above rule, the 3D conformations were generated, and then we can construct the hypothesis model by using "Ligand Pharmacophore Mapping protocol”.

\section{Quality Validation Methods of PhModels}

Cost-function Analysis, there are two important theoretical cost values (in units of bits) which were used to determine each PhModel. One is the fixed cost that represents the simplest model that accurately predict all experimental $\mathrm{IC}_{50}$ values of training set inhibitors. The other one is the null cost which represents the largest cost for a model that has no prediction ability. A good PhModel should content the following condition. The difference between the fixed and 
null costs is $>70$ bits that means there is a perfect chance of $>90 \%$ that the experimental and estimated activities will be correlation. In addition, the total cost should be close to the fixed cost, and the configuration cost, representing the space complexity of models, must be $<17$ bits for a good model.

Fischer's Cross-validation Test was used to validate the quality of PhModels, and to verify the structure and activity of the models whether has a great relativity. The $\mathrm{IC}_{50}$ values of training set inhibitors were randomly assigned to members of the set, and this set will be used to generate randomly PhModels. The same features and parameters used to generate the ten initial PhModels were incorporated into this trial. The formula of Fischer's cross-validation test is written as:

$$
\text { Confidence }(\%)=[1-(1+x) /(1+y)] \times 100 \%
$$

where $x$ is the number of the hypotheses which have a total cost lower than the original hypotheses, and $y$ is the number of times the procedure executed. According to the formula, we can run 19 times to achieve a confidence level of $95 \%$.

Correlation Coefficient Analysis was used to verify the relativity of estimated $\mathrm{IC}_{50}$ and experimental $\mathrm{IC}_{50}$ for the training and testing set inhibitors. If the value of the correlation coefficient is close to 1 , the verified $\mathrm{PhModel}$ will be good.

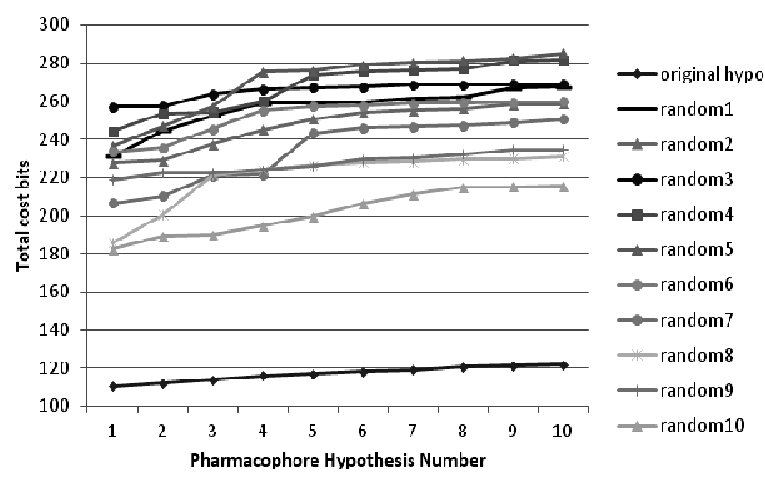

Fig. 1. The original total cost of PhModel and ten random total costs of lowest ones by randomized pharmacophore.

\section{RESULTS}

\section{A. PhModel Generation Results}

Ten PhModels were generated by using 25 training set inhibitors. Each of them took four features: three hydrogen bond acceptors (A) and one hydrophobic aromatic (HYAR). The characteristics of ten PhModels, Hypo01 to Hypo10, were listed in Table II. The null cost of ten established models is 304.19 bits, and the fixed cost is 89.77 bits. In addition, the difference between null and fixed cost is 214.42 bits which means all ten hypotheses had high prediction ability. In other words, a difference should be $>70$ bits then there is an excellent chance of $>90 \%$ that the experimental and estimated activities will be correlation. Furthermore, the configuration cost is 16.07 , which is in allowed range lower than the value of 17 bits. According to the cost function analysis, the total cost of a good quality PhModel should approach the fixed cost and be far away from the null cost. In the results, the highest total cost of worst hypothesis, Hypo10, is 125.89 bits. The correlation coefficient $(r)$ of the ten hypotheses is ranged from 0.955 to 0.92 .

\section{B. Fischer's Cross-Validation Test}

Fischer's test is used to cross-validate the quality of PhModels. The $\mathrm{IC}_{50}$ values of training set inhibitors were randomly assigned to each inhibitor in the training set. The parameters of randomized PhModel were the same as those used to generate the original PhModel. In total, 19 random PhModels were generated. The total cost of the ten original PhModel all were less than any of the 19 random PhModel, and it indicated that the ten PhModels had a 95\% confidence level with the achieved validation to generate the training set inhibitors. Fig. 1 shows the total costs from original PhModels and the ten lowest total costs from randomization. As the above, the training set inhibitors could not be generated by randomized PhModels.

\section{Correlation Analysis of Testing Set Inhibitors}

Another main goal is to evaluate the prediction ability of a PhModel in testing set inhibitors. Here, the prediction activities of 133 inhibitors in the testing set were estimated by ten hypotheses. The correlation coefficient $\left(r_{\text {test }}\right)$ was obtained from the linear regression of estimated activity versus the experimental. In the results, the best $r_{\text {test }}$ for testing set inhibitors was 0.81 obtained from Hypo01 shown in Table II. We also compared with the other Hypo models that Hypo01 had the best ability to predict the experimental activity. Therefore, we considered that Hypo01 was a dependable predictor of Chk2 inhibitor activity.

\section{REFERENCES}

[1] K. M. Culligan, C. E. Robertson, J. Foreman, P. Doerner, and A. B. Britt, "ATR and ATM play both distinct and additive roles in response to ionizing radiation," The Plant Journal, vol. 48, pp. 947-961, 2006.

[2] J. Yang, Z. P. Xu, Y. Huang, H. E. Hamrick, P. J. Duerksen-Hughes, and Y. N. Yu, "ATM and ATR: Sensing DNA damage," World J Gastroenterol, vol. 10, no. 2, pp. 155-160, 2004.

[3] Q. Liu, S. Guntuku, X. S. Cui, et al., "Chk1 is an essential kinase that is regulated by Atr and required for the G 2/M DNA damage checkpoint," Genes Dev., vol. 14, pp. 1448-1459, 2000.

[4] C. T. Alveal, T. M Calonge, and M. J O'Connell, "Regulation of Chk1," Cell Division, vol. 4, pp. 8, 2009.

[5] J. Bartek, J. Falck, and J. Lukas, "CHK2 KINASE - A BUSY MESSENGER,” Nat Rev Mol Cell Biol., vol. 2, pp. 877-86, Dec, 2001.

[6] J. Li, B. L. Williams, L. F. Haire, M. Goldberg, E. Wilker, D. Durocher, M. B. Yaffe, S. P. Jackson, and S. J. Smerdon, "Structural and functional versatility of the FHA domain in DNA-damage signaling by the tumor suppressor kinase CHK2," Molecular Cell, vol. 9, pp. 1045-1054, May, 2002.

[7] A. W. Oliver, A. Paul, K. J. Boxall, S. E. Barrie, G. W. Aherne, M. D. Garrett, S. Mittnacht, and L. H. Pearl, "Trans-activation of the DNA-damage signalling protein kinase Chk2 by T-loop exchange," The EMBO Journal, vol. 25, pp. 3179-3190, 2006.

[8] Z. Cai, N. H. Chehab, and N. P. Pavletich, "Structure and activation mechanism of the CHK2 DNA damage checkpoint kinase," Molecular Cell, vol. 35, pp. 818-829, September, 2009.

[9] Z. A. Stewart and J. A. Pietenpol, "Signaling and cell cycle checkpoints," Chem. Res. Toxicol., vol. 14, no. 3, pp. 53, 2001.

[10] G.T. Lountos, A. G. Jobson, J. E. Tropea, C. R. Self, G. Zhang, Y. Pommier, R. H. Shoemaker, and D. S. Waugh, "Structural characterization of inhibitor complexes with checkpoint kinase 2 (Chk2), a drug target for cancer therapy," Journal of Structural Biology, vol. 176, pp. 292-301, 2011.

[11] A. G. Jobson, G. T. Lountos, P. L. Lorenzi, J. Llamas, J. Connelly, D. Cerna, J. E. Tropea, A. Onda, G. Zoppoli, S. Kondapaka, G. Zhang, N. 
J. Caplen, J. .H. Cardellina II, S. S. Yoo, A. Monks, C. Self, D. S. Waugh, R. H. Shoemaker, and Y. Pommier, "Cellular inhibition of Checkpoint Kinase 2 (Chk2) and potentiation of camptothecins and radiation by the novel Chk2 inhibitor PV1019 [7-Nitro-1H-indole-2-carboxylic acid \{4-[1-(guanidinohydrazone)-ethyl]-phenyl\}-amide]," JPET, vol. 331, pp. 816-826, 2009.

[12] J. J. Caldwell, E. J. Welsh, C. Matijssen, V. E. Anderson, L. Antoni, K. Boxall, F. Urban, A. Hayes, F. I. Raynaud, L. J. M. Rigoreau, T. Raynham, G. W. Aherne, L. H. Pearl, A. W. Oliver, M. D. Garrett, and I. Collins, "Structure-based design of potent and selective 2-(Quinazolin-2-yl) phenol Inhibitors of Checkpoint Kinase 2," J. Med. Chem., vol. 54, pp. 580-590, 2011.

[13] G. T. Lountos, A. G. Jobson, J. E. Tropea, C. R. Self, G. Zhang, Y. Pommier, R. H. Shoemaker, and D. S. Waugh, "X-ray structures of checkpoint kinase 2 in complex with inhibitors that target its gatekeeper-dependent hydrophobic pocket," FEBS Letters, vol. 585, pp. 3245-3249, 2011.

[14] S. Hilton, S. Naud, J. J. Caldwell, K. Boxall, S. Burns, V. E. Anderson, L. Antoni, C. E. Allen, L. H. Pearl, A. W. Oliver, G. W. Aherne, M. D. Garrett, and I. Collins, "Identification and characterisation of 2-aminopyridine inhibitors of checkpoint kinase 2," Bioorganic \& Medicinal Chemistry, vol. 18, pp. 707-718, 2010.

[15] H. Kubinyi, G. Folkers, and Y. C. Martin, "3D QSAR in drug design recent advances," vol. 3, pp. 1-353, 2002.

[16] Y. K. Jiang, "Molecular docking and 3D-QSAR studies on $\beta$-phenylalanine derivatives as dipeptidyl peptidase IV inhibitors," $J$ Mol Model, vol. 16, pp. 1239-1249, 2010.

[17] R. R. S. Pissurlenkar, M. S. Shaikh, and E. C. Coutinho, "3D-QSAR studies of Dipeptidyl peptidase IV inhibitors using a docking based alignment," NVPJ Mol Model, vol. 13, pp. 1047-1071, 2007.

[18] T. Puzyn et al., "3D-QSAR - APPLICATIONS, RECENT ADVANCES, AND LIMITATIONS," Recent Advances in QSAR Studies, pp. 103-125, 2010.

[19] A. Lauria, M. Ippolito, M. Fazzari, M. Tutone, F. D. Blasi, Francesco Mingoia, and A. M. Almerico, "IKK-inhibitors: An analysis of drug-receptor interaction by using molecular docking and pharmacophore 3D-QSAR approaches," Journal of Molecular Graphics and Modelling, vol. 29, pp. 72-81, 2010.

[20] S. John, S. Thangapandian, M. Arooj, J. C. Hong, K. D. Kim, and K. W. Lee, "Development, evaluation and application of 3D QSAR pharmacophore model in the discovery of potential human renin inhibitors," BMC Bioinformatics, vol. 12, Suppl 14, pp. 1-14, 2011.

[21] K. W. Lee and J. M. Briggs, "Comparative molecular field analysis (CoMFA) study of epothilones - tubulin depolymerization inhibitors: Pharmacophore development using 3D QSAR methods," Journal of Computer-Aided Molecular Design, vol. 15, pp. 41-55, 2001.

[22] F. A. Pasha, M. Muddassar, and S. J. Cho, "Molecular docking and 3D QSAR studies of Chk2 inhibitors," Chem Biol Drug Des, vol. 73, pp. 292-300, 2009.

[23] S. Durdagi, T. Mavromoustakos, and M. G. Papadopoulos, "3D QSAR CoMFA/CoMSIA, molecular docking and molecular dynamics studies of fullerene-based HIV-1 PR inhibitors," Bioorganic \& Medicinal Chemistry Letters, vol. 18, pp. 6283-6289, 2008.

[24] M. E. Suh, S. Y. Park, and H. J. Lee, "Comparison of QSAR methods (CoMFA, CoMSIA, HQSAR) of anticancer 1-N-substituted Imidazoquinoline-4, 9-dione derivatives. Bull," Korean Chem. Soc., vol. 23, no. 3, pp. 417-422, 2002.

[25] S. J. Bang and S. J. Cho, "Comparative molecular field analysis (CoMFA) and comparative molecular similarity index analysis (CoMSIA) study of mutagen X. bull," Korean Chem. Soc., vol. 25, no. 10, pp. 1525-1530, 2004.

[26] L. Ghemtio, Y. Zhang, and H. Xhaard, "CoMFA/CoMSIA and pharmacophore modelling as a powerful tools for efficient virtual screening: Application to anti-leishmanial betulin derivatives," Centre for Drug Research, Faculty of Pharmacy, University of Helsinki, Finland, 2012.

[27] G. Klebe, "Comparative molecular similarity indices analysis: CoMSIA," Institute of Pharmaceutical Chemistry, University of Marburg, Marbucher Weg 6, D 35032 Marburg, Germany, 2002.

[28] G. Klebe and U. Abraham, "Comparative molecular similarity index analysis (CoMSIA) to study hydrogen-bonding properties and to score combinatorial libraries," Journal of Computer-Aided Molecular Design, vol. 13, pp. 1-10, 1999.

[29] M. Gupta, S. Gupta, H. Dureja, and A. K. Madan, "Superaugmented eccentric distance sum connectivity indices: Novel highly discriminating topological descriptors for QSAR/QSPR," Chem Biol Drug Des, vol. 79, pp. 38-52, 2012.

[30] I. Mitra, A. Saha, and K. Roy, "Pharmacophore mapping of arylamino-substituted benzo[b]thiophenes as free radical scavengers," J Mol Model, vol. 16, pp. 1585-1596, 2010.

[31] K. Boppana, P. K. Dubey, S. A. R. P. Jagarlapudi, S. Vadivelan, and G. Rambabu, "Knowledge based identification of MAO-B selective inhibitors using pharmacophore and structure based virtual screening models," European Journal of Medicinal Chemistry, vol. 44, pp. 3584-3590, 2009.

[32] M. Chopra, R. Gupta, S. Gupta, and D. Saluja., "Molecular modeling study on chemically diverse series of cyclooxygenase-2 selective inhibitors: generation of predictive pharmacophore model using Catalyst," J Mol Model, vol. 14, pp. 1087-1099, 2008.

[33] A. Gaulton, L. J. Bellis, A. P. Bento, J. Chambers, M. Davies, A. Hersey, Y. Light, S. McGlinchey, D. Michalovich, B. Al-Lazikani, and J. P. Overington, "ChEMBL: a large-scale bioactivity database for drug discovery," Nucleic Acids Research, vol. 40, Database issue, D1100-D1107, 2012

[34] P. W. Sprague, "Automated chemical hypothesis generation and database searching with Catalyst(R)," Perspect. Drug Discovery Des, vol. 3, pp. 1-20, 1995.

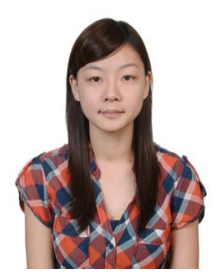

Yen-Ling Wang received a B.S. degree in Department of Computer Science and Information Engineering from Fu Jen University in 2009. She is currently a Master student in the Department of Computer Science and Information Engineering at Chang Gung University. His research interests are in the areas of Computational Chemistry and Viral Research.

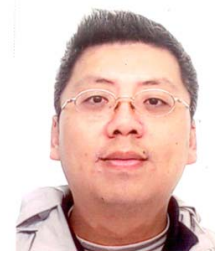

Chun-Yuan Lin received a B.S. degree in Department of Information Engineering and Computer Science from Feng Chia University in 1999, and the M.S. and Ph.D. degrees in Department of Information Engineering and Computer Science from Feng Chia University in 2000 and 2003, respectively. He joined the Institute of Molecular and Cellular Biology and the Department of Computer Science at National Tsing Hua University as a post-doctoral fellow in 2003 and 2006, respectively. In 2007, he joined the Department of Computer Science and Information Engineering at Chang Gung University as an assistant professor. He also is a faculty Member at Research center for Emerging Viral Infections in Chang Gung University. His research interests are in the areas of parallel and distributed computing, proteomics, Genomics, Systems Biology, Next-Generation Sequencing and Computational Chemistry.

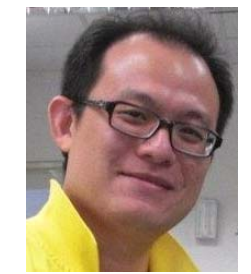

Kuei-Chung Shih received a B.S. degree in Department of Information Management from Nan Kai University of Technology in 2003, and the M.S. degrees in Department Institute of Bioinformatics from Asia University in 2005. In 2011, he received a Ph.D. degree in Department of Computer Science from National Tsing Hua University. He joined the Department of Computer Science at National Tsing Hua University as a post-doctoral fellow in 2011.His research interests are in the areas of bioinformatics, target drug design, pharmacophore, 3D-QSAR, molecular dynamics and computational chemistry.

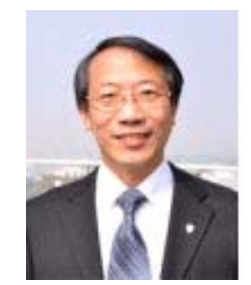

Chuan Yi Tang received his B.S. degree in Electrical Engineering and M.S. degree in Computer Science from National Tsing Hua University, Taiwan, in 1980 and 1982, respectively. $\mathrm{He}$ obtained his $\mathrm{Ph} . \mathrm{D}$. from the Department of Computer Science and Information Engineering at National Chiao Tung University, Taiwan, in 1985. In the same year, he joined the

faculty of Computer Science at National Tsing Hua University, where he became a full professor in 1992. Currently, he is the President of the Providence University, Taiwan. His research interests include the analysis and design of algorithms, computational molecular biology, bioinformatics, parallel processing, and computer aided engineering. More than fifty research papers have been published in the prestigious journals of computer science and related fields. 